\title{
Coriolus versicolor polysaccharides (CVP) regulates neuronal apoptosis in cerebral ischemia-reperfusion injury via the p38MAPK signaling pathway
}

\author{
Lei $\mathrm{Li}^{1}$, Yan $\mathrm{Li}^{2}$, Cheng Miao ${ }^{1}$, Yi Liu ${ }^{1}$, Rui Liu ${ }^{1}$ \\ ${ }^{1}$ Department of Neurology, Xinxiang Central Hospital, Xinxiang, China; ${ }^{2}$ Clinical Medical College of Tianjin Medical University, Tianjin, China \\ Contributions: (I) Conception and design: L Li, R Liu; (II) Administrative support: Y Li; (III) Provision of study materials or patients: L Li, Y Li, C \\ Miao, Y Liu; (IV) Collection and assembly of data: All authors; (V) Data analysis and interpretation: L Li, C Miao, Y Liu, R Liu; (VI) Manuscript \\ writing: All authors; (VII) Final approval of manuscript: All authors. \\ Correspondence to: Rui Liu. Xinxiang Medical University, 601 Jinsui Avenue, Xinxiang, China. Email: sandy_lr11698@126.com.
}

\begin{abstract}
Background: This study aims to investigate the regulation of herbal polysaccharide, Coriolus versicolor polysaccharides (CVP), on neuronal apoptosis in a rat cerebral ischemia-reperfusion injury (CIRI) model. We also intend to explore the mechanisms and effectiveness of CVP in the treatment of neuronal apoptosis in CIRI rats, including neurological function, cerebral infarction volume, inflammatory factors, and the p38 mitogen-activated protein kinase (p38MAPK) signaling pathway as well as its downstream protein cleavedCaspase-3.
\end{abstract}

Methods: A CIRI model was established in rats using the Longa method of middle cerebral artery occlusion. Neurological function scores and cerebral infarction volumes were measured in CIRI rats. Annexin V-fluorescein isothiocyanate (FITC) were used to measure neuronal apoptosis in CIRI rats. The levels of interleukin- $1 \beta$ (IL-1 $\beta$ ) and tumor necrosis factor- $\alpha$ (TNF- $\alpha$ ) in CIRI rats were determined using enzymelinked immunosorbent assay (ELISA). A western blot assay was used to measure the protein expression levels of p38MAPK, phospho-p38MAPK (p-p38MAPK), Bcl-2, Bax and cleaved-Caspase-3 in brain tissue of CIRI rats.

Results: CVP can effectively improve the neurological function of rats after 6, 12, 24, and 48 h of CIRI. It can also improve the behavioral test, reduce the cerebral infarction volume and inhibit the apoptosis of nerve cells in CIRI rats. The protein expression levels of p-p38MAPK and cleaved-Caspase-3 exhibited a decreasing trend following CVP administration.

Conclusions: CVP can significantly reduce the pathological characteristics of CIRI in rats and inhibit the apoptosis of nerve cells around the lesions. The mechanism of its effectiveness is related to inhibiting the activation of the p38MAPK signaling pathway.

Keywords: cerebral ischemia-reperfusion injury; Coriolus versicolor polysaccharides (CVP); p38 mitogenactivated protein kinase (p38MAPK); apoptosis

Submitted Jul 16, 2020. Accepted for publication Sep 08, 2020.

doi: 10.21037/atm-20-5759

View this article at: http://dx.doi.org/10.21037/atm-20-5759

\section{Introduction}

Ischemic cerebrovascular disease is a common neurological condition characterized by high rates of prevalence, disability, and mortality. It is the third leading cause of death and permanent disability in adults worldwide (1).
Reperfusion is currently the most effective way to clear the cerebral vascular infarction and restore blood supply to the ischemic part of the brain (2). However, cerebral ischemiareperfusion is also very likely to cause injury to brain tissue [known as cerebral ischemia-reperfusion injury (CIRI)]. 
CIRI is a physiological and pathological process involving multiple mechanisms. Due to ischemia and hypoxia, free radicals are rapidly formed in brain tissue following blood reperfusion, resulting in the release of a large number of inflammatory factors and the overload of intracellular calcium. This in turn activates apoptotic genes, ultimately leading to neuronal apoptosis (3). Neuronal apoptosis is known to be associated with a series of inflammatory and apoptotic factors, and signal transduction pathways, though, the specific regulatory mechanisms are still poorly understood. Currently, the process of inducing and regulating neuronal apoptosis is in the exploratory stage. Therefore, understanding the mechanisms of neuronal apoptosis through the investigation of polysaccharide drugs with therapeutic effects on CIRI is critically important for reducing brain tissue damage and improving brain function following CIRI.

Till date, it was reported that plenty of polysaccharides from traditional Chinese medicines exhibited protection effect on ischemia-reperfusion injury (4). The mechanisms involved were mainly regulating endogenous antioxidants, improving inflammation response and minimizing infarction size. Here, we introduced a new agent named Coriolus versicolor (L.ex Fr.) Quel, which is a medicinal fungus traditionally used in Chinese medicine. It contains more than 20 different compounds including organic acids, triterpenoids, alkaloids, steroids, proteins, and polysaccharides, among which, Coriolus versicolor polysaccharide (CVP) is the major component. CVP is extracted from the fruit body of Coriolus versicolor and has various biopharmacological functions such as immune system regulation, and anti-tumor, antibacterial, and antiviral activity $(5,6)$. Studies have shown that CVP has an effective therapeutic role in the treatment of cerebral ischemic diseases, though there remains a lack of research regarding its associated mechanisms (7).

The p38 mitogen-activated protein kinase (p38MAPK) is composed of more than 300 amino acids and is an important member of the mitogen-activated protein kinase (MAPK) family. Studies have shown that the p38MAPK signaling pathway plays a significant role in oxidative stress, inflammatory response, and neuronal apoptosis (8). After activation, p38MAPK eventually leads to neuronal death by regulating apoptosis-related proteins: Caspase-3, Caspase-9, and apoptosis regulator B-cell lymphoma 2 (Bcl-2)/Bcl2 -associated X (Bax). Studies conducted by Su et al. have demonstrated that activation of the p38MAPK signaling pathway in ischemic diseases can induce the expression of proapoptotic protein caspase-3, which ultimately leads to apoptosis (9). Therefore, in this study, we treated the rat CIRI model with CVP to investigate the effects of CVP on CIRI lesions and on changes in p38MAPK and cleavedCaspase-3 protein expression. We also aimed to explore the mechanism of CVP in the inhibition of neuronal apoptosis in CIRI.

We present the following article in accordance with the ARRIVE reporting checklist (available at http://dx.doi. org/10.21037/atm-20-5759).

\section{Methods}

\section{Animal models and grouping}

A total of 216 healthy male Sprague Dawley (SD) rats $(280 \pm 30 \mathrm{~g})$ were used as subjects, and were purchased from the Shanghai Slack Laboratory Animal Co. Ltd. Rats were initially placed in the standard feeding conditions with natural light. After a week of adaptive feeding, they were randomly divided into sham, CIRI, CVP groups and Anisomycin (ANI, p38 agonist) group with 54 rats in each group. Each group was then divided into 6, 12, 24, and $48 \mathrm{~h}$ groups according to different times after cerebral ischemiareperfusion.

This study was approved by Xinxiang Central Hospital (ZXYY-KY-0124). During our experiments, we strictly follow the Regulations on the Administration of Experimental Animals, The Guiding Opinions on treating Experimental Animals well and other relevant laws and regulations.

\section{CVP preparation and purification}

The wild Coriolus versicolor fruit body was pulverized and sieved, heat soaked with distilled water at a ratio of 1:50, and extracted three times under reflux. The supernatant was collected and the bound substance was eluted away with a diethylaminoethyl (DEAE)-sephacel $(2.6 \times 100 \mathrm{~cm})$ anion exchange chromatography column and a $0-2 \mathrm{~mol} / \mathrm{L}$ linear gradient sodium chloride $(\mathrm{NaCl})$ solution. The total polysaccharide content in the product was determined using the phenol-sulfuric acid method. The polysaccharides were then precipitated three times with absolute ethanol, and the precipitate was dried under reduced pressure at $60{ }^{\circ} \mathrm{C}$ for $4 \mathrm{~h}$ to obtain purified polysaccharides. The glucan content in the final product was $96.9 \%$, with negligible protein and nucleic acid contamination (absorbance at 280 and $260 \mathrm{~nm}$ 
were approximately zero).

\section{High-performance liquid chromatography (HPLC) analysis}

The purified CVP samples were analyzed with HPLC using a TSKgel G4000PW $\mathrm{XL}_{\mathrm{XL}}$ column and an evaporative lightscattering detector (10). Using water as the mobile phase, $10 \mathrm{~mL}$ of the sample solution $(1 \mathrm{mg} / \mathrm{mL})$ was taken at a flow rate of $1 \mathrm{~mL} / \mathrm{min}$. Dextran standards [molecular weight (MW): 1,000, 2,000, 4,000, 70,000, 100,000 Dalton (Da)] (Yease, China) were used for the calibration of the linear regression curve.

\section{Preparation of the CIRI model in rats}

The CIRI model was established in the rats according to the Longa method of middle cerebral artery occlusion. Initially, rats were fasted for 12 hours before surgery but were free to drink water. Before the operation, rats were weighed and anesthetized intraperitoneally with $3 \%$ pentobarbital sodium at $40 \mathrm{mg} / \mathrm{kg}$ and then placed on the operating table. The middle of the neck was routinely disinfected and a $2 \mathrm{~cm}$ longitudinal incision was made. The right common carotid artery, internal carotid artery, and external carotid artery were separated and exposed. Next, the branch of the external carotid artery and the pterygoid artery were ligated at the bifurcation position of the internal carotid artery and the external carotid artery. The proximal end of the common carotid artery near the heart was ligated with a nylon fishing line. A small opening was made at the distal end of the ligature to facilitate insertion of the nylon fishing line into the middle cerebral artery through the internal carotid artery to induce cerebral ischemia via middle cerebral artery occlusion. The extravascular portion of the tying line was ligated and fixed. After 2 hours of ischemia, the suture was removed to restore blood supply (reperfusion). The success of the model was confirmed by the fact that (when awake) the rats exhibited slow movement and tail-lifting, they flexed and adducted their left front limbs, and when they crawled, they turned left. This procedure was performed in the same way for the sham and model groups, except that the nylon fishing line was not inserted into the middle cerebral artery in the sham group. The CVP group was intraperitoneally injected with a CVP solution $30 \mathrm{~min}$ before the reperfusion according to body weight $(150 \mathrm{mg} / \mathrm{kg} / \mathrm{d})$. The CIRI and intervention groups received the same amount of normal saline in the intraperitoneal injection. The ANI group received intraperitoneally injection of CVP solution $30 \mathrm{~min}$ prior to anisomycin $(100 \mu \mathrm{g} / \mu \mathrm{L}$ dissolved in $10 \mathrm{~N} \mathrm{HCl})$ injection.

\section{Neurological function score}

Neurological symptoms were observed in each group following cerebral ischemia-reperfusion, and neurological function was measured using the Longa scoring method. The standard was: zero points for rats that exhibited no neurological injury and whose activity was normal; one point for rats who could not fully extend their contralateral forelimb; two points for rats who circle to the opposite side; three points for rats who tilted to the opposite side; four points for rats who could not be self-issued and lose consciousness.

\section{Measurement of cerebral infarct size}

Rats were sacrificed 24 hours after ischemia-reperfusion and a craniotomy was performed. The brain was stored in a $-4{ }^{\circ} \mathrm{C}$ refrigerator for $20 \mathrm{~min}$ and then divided into five aliquots of coronary sections, which were then placed in a $2 \%$ 2,3,5-triphenyl-2H-tetrazolium chloride (TTC) solution in a $37{ }^{\circ} \mathrm{C}$ water bath and fixed with $4 \%$ paraformaldehyde. After TTC staining, normal brain tissue showed a pink color, and brain tissue in the infarct area showed a pale white color. The infarct volume of each brain slice was measured using Image $\mathrm{J}$ image analysis software, and was calculated as: infarct volume $\left(\mathrm{mm}^{3}\right)=$ infarct size $x$ thickness/2.

\section{Beam walking test}

Rats were trained three days before injury on beam walking. The beam had a length of $1,390 \mathrm{~mm}$ and a $20 \mathrm{~mm}$ width. Time to across the beam and footfalls were evaluated to assess the motor coordination and balance.

\section{Forelimb placement assay}

Forelimb motor activity was monitored to detect the neuronal activity of rats. About $0.5 \mathrm{~cm}$ away from the edge of the experimental table, the experimenter brushed the whiskers on each side gently. The undamaged rats would immediately place their forelimbs on the desktop, and the rats with nerve function impairment would fail to complete their actions. The rats were tested 10 times on each side, 
and the percentage of times their front limbs touched the table was the score of that side.

\section{Detection of apoptosis}

Apoptotic cells were analyzed using Annexin V-fluorescein isothiocyanate (FITC) flow cytometry. Fresh brain tissue from each group was ground to homogenate at $4{ }^{\circ} \mathrm{C}$, then filtered through a 300 mesh screen. Phosphate buffered saline (PBS) was used to prepare a cell suspension to be counted. Then $1 \times 10^{7}$ cells were taken from each group of samples and centrifuged at 1,000 rpm for $5 \mathrm{~min}$. The supernatant cells were then washed with $1 \mathrm{~mL}$ PBS buffer. They were subsequently centrifuged again at 1,000 rpm for 5 minutes, and washed twice with PBS. Five hundred $\mu \mathrm{L}$ of annexin binding buffer was added to the pellet and mixed by pipetting. After this, $5 \mu \mathrm{L}$ of Annexin V-FITC reagent was added and the final solution was kept still for $10 \mathrm{~min}$ at room temperature in the dark. Next, $10 \mu \mathrm{L}$ of propidine iodide $(\mathrm{PI})$ reagent was added at room temperature and incubated for $5 \mathrm{~min}$ in the dark. The fluorescence intensity of each group of cells was measured by flow cytometry, and the percentage of apoptotic cells was calculated.

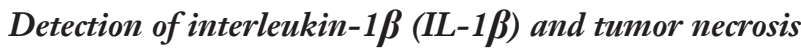 factor- $\alpha(T N F-\alpha)$ levels}

Rats were sacrificed $24 \mathrm{~h}$ after cerebral ischemiareperfusion. Brain tissue was extracted from the cerebral infarction and a $10 \%$ tissue homogenate was prepared and centrifuged for $10 \mathrm{~min}$. IL-1 $\beta$ and $\mathrm{TNF}-\alpha$ were measured using an enzyme-linked immunosorbent assay (ELISA) kit [purchased from R\&D System (USA)] according to the manufacturer's instructions.

\section{Detection of phospho-p38MAPK (p-p38MAPK) and cleaved-Caspase-3 protein expression by western blot}

Twenty-four h after cerebral ischemia-reperfusion, the appropriate amount of brain tissue from each group was placed in a cell lysate (Beyotime, China) to extract total protein. The protein concentration was determined using a bicinchoninic acid (BCA) assay kit (Beyotime, China). Next, a $5 \times$ sodium dodecyl sulfate-polyacrylamide gel electrophoresis (SDS-PAGE) loading buffer was mixed with the sample protein and heated in a $95-97{ }^{\circ} \mathrm{C}$ water bath for $5 \mathrm{~min}$, and then stored in a $-80{ }^{\circ} \mathrm{C}$ refrigerator for the subsequent experiment. The quantified amount of protein was loaded and separated by $12 \%$ PAGE, and a polyvinylidine difluoride (PVDF) membrane was used to transfer the protein. $5 \%$ skim milk was used to block at room temperature for $1 \mathrm{~h}$, and anti-phosphorylated (p)p38MAPK (1:3,000, Cell Signaling Technology, USA), anti-cleaved caspase-3 (1:2,000, Santa Cruz Biotechnology, USA), and $\beta$-actin (1:1,000, Santa Cruz Biotechnology, USA) were added and incubated overnight at $4{ }^{\circ} \mathrm{C}$. The second antibody was added and incubated for $2 \mathrm{~h}$ at room temperature the following day. After washing the membrane with tris-buffered saline, $0.1 \%$ Tween 20 (TBST), Image Lab 3.0 was used for imaging. Image J software was used to quantitatively analyze the difference in protein expression in each group. $\beta$-actin was used as an internal reference for the quantitative measurement of protein expression.

\section{Statistical analysis}

Data analysis was performed using SPSS 22.0 statistical software and OriginPro 8. The measurements were expressed as mean \pm standard deviation. For comparison between two groups, two independent sample $t$-tests were used when the variance was uniform, and the Satterthwaite approximate $t$-test was used when the variance was not uniform. One-way analysis of variance (ANOVA) was used for comparison between the groups. $\mathrm{P}<0.05$ was considered statistically significant.

\section{Results}

\section{Effect of CVP on neurological function in rats with cerebral ischemia-reperfusion}

The CVP solution was purified by a series of DEAEsephacel anion exchange chromatography columns and analyzed using HPLC to obtain a CVP solution with a single peak. According to its retention time, the MW of CVP is $1,820 \mathrm{kDa}$ (Figure 1A). To determine the effect of CVP on neurological function in rats with cerebral ischemia-reperfusion, we measured neurological functions in the sham, CIRI, and CVP groups at 6, 12, 24, and $48 \mathrm{~h}$ after cerebral ischemia-reperfusion. The results are shown in Figure 1B. The neurological function of the sham group was normal, with a score of zero. The neurological scores of the CIRI group at $6,12,24$, and $48 \mathrm{~h}$ were $2.3,3.5$, 3.6 , and 3.2, respectively. For the same time periods, the neurological function scores of the CVP group were: 0.9, 
A

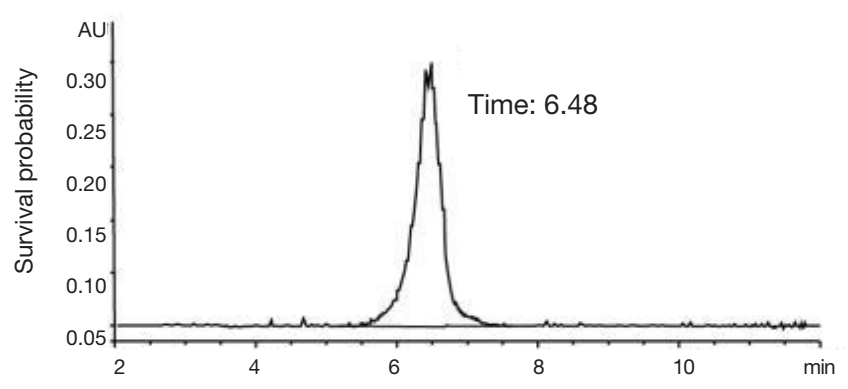

B

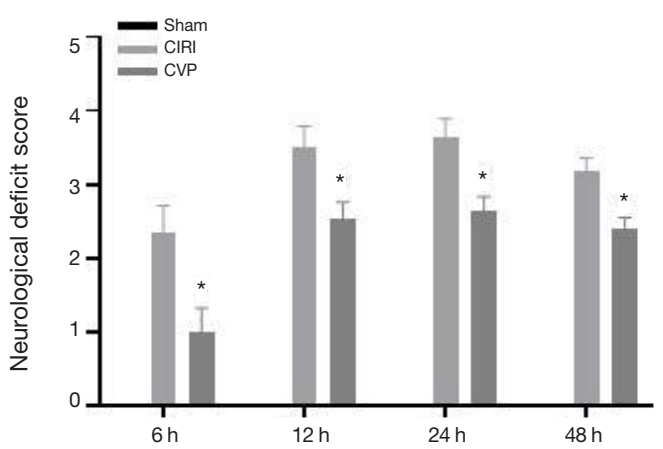

Figure 1 Structural analysis of CVP and the effect of CVP on neurological function in rats with cerebral ischemia-reperfusion at different time points. (A) HPLC analysis of CVP. (B) Neurological function scores of cerebral ischemia-reperfusion rats in different experimental groups at different time points. *, indicates compared to the CIRI group, $\mathrm{P}<0.05$. CIRI, cerebral ischemia-reperfusion injury; CVP, coriolus versicolor polysaccharides; ANI, anysomycin; HPLC, high-performance liquid chromatography.

2.5, 2.6, and 2.4, respectively. All rats in the CIRI and CVP groups showed significant symptoms of neurological impairment. Nevertheless, compared with the CIRI group, the neurological function of the rats in the CVP group was significantly restored, and the difference between the two groups was statistically significant $(\mathrm{P}<0.05)$. These results indicated that CVP can effectively alleviate the neurological dysfunction in CIRI rats.

\section{Effect of CVP on cerebral infarction volume in rats with cerebral ischemia-reperfusion}

The brain infarct volume of the sham, CIRI, CVP and ANI groups was determined using the TTC method. The results are shown in Figure 2. Compared with the sham group, the cerebral infarction volume of the rats in the CIRI group was markedly increased at $6,12,24$, and $48 \mathrm{~h}$ after cerebral ischemia-reperfusion, and the difference was statistically significant $(\mathrm{P}<0.001)$. The CVP group had a lower cerebral infarction volume than the CIRI group at each time point and the difference was statistically significant $(\mathrm{P}<0.01)$, indicating that CVP may have a certain degree of therapeutic effect on cerebral infarction tissue after cerebral ischemia-reperfusion.

\section{Effect of CVP on neuronal apoptosis in rats with cerebral ischemia-reperfusion}

The Annexin V-FITC flow cytometry (Figure 3) were used to detect neuronal apoptosis in the cerebral infarction of rats in each group at different time points. The results showed that the number of positive cells in the CIRI group was significantly greater than that in the sham and ANI group, and the difference was statistically significant $(\mathrm{P}<0.001)$. Compared with the CIRI group, the number of positive cells in the CVP group was significantly reduced $(\mathrm{P}<0.05)$. The decrease in the number of positive cells in the CVP group and the decrease in the apoptotic rate further highlighted the therapeutic effect of CVP on CIRI rats.

\section{Effect of CVP on behavioral test}

As shown in Figure 4, rats in CIRI group had decreased scores in beam walking test and forelimb placement test when compared to sham group $(\mathrm{P}<0.001)$. Treatment of CVP significantly improved behavioral functional score in rats, compared to CIRI group $(\mathrm{P}<0.05)$. No significance was found between ANI and CIRI group.

\section{The effect of CVP on the levels of IL-1 $\beta$ and TNF- $\alpha$ in rats with cerebral ischemia-reperfusion}

Cerebral ischemia is typically followed by inflammation. We measured the levels of IL- $1 \beta$ and TNF- $\alpha$ levels in the sham, CIRI, CVP and ANI groups $24 \mathrm{~h}$ after cerebral ischemiareperfusion. As shown in Figure 5, IL- $1 \beta(\mathrm{P}<0.001)$ and TNF- $\alpha(\mathrm{P}<0.01)$ levels in the CIRI group were considerably higher than those in the sham and ANI group, and the differences were statistically significant. Also, the levels of IL-1 $\beta$ and TNF- $\alpha$ in the CVP group were notably lower 


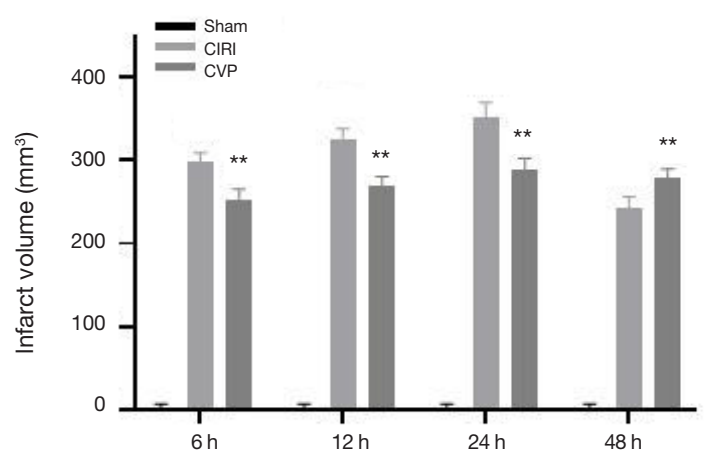

Figure 2 Effect of CVP on cerebral infarction in rats with cerebral ischemia-reperfusion at different time points. ${ }^{* *}$, comparison with the CIRI group, $\mathrm{P}<0.001$. CIRI, cerebral ischemia-reperfusion injury; CVP, coriolus versicolor polysaccharides; ANI, anysomycin.

than those in the CIRI group $(\mathrm{P}<0.01)$ suggesting that an inflammatory reaction occurred after cerebral ischemiareperfusion, and that CVP reduced the expression of inflammatory factors.

\section{Effects of CVP on the expression of Bcl-2, Bax, p-p38MAPK, cleaved-Caspase-3 protein in rats with cerebral ischemia-reperfusion}

We examined the expression of Bcl-2, Bax, p-p38MAPK and cleaved-Caspase- 3 protein using western blotting, as shown in Figure 6. The expression of these proteins was substantially higher in the CIRI group than in the sham and ANI group $(\mathrm{P}<0.001)$. Also, the expression of $\mathrm{p}-\mathrm{p} 38 \mathrm{MAPK}$ $(\mathrm{P}<0.05)$ and cleaved-Caspase- $3(\mathrm{P}<0.001)$ in the CVP group was significantly reduced compared with the CIRI group. These results indicated that CVP may regulate the effect of the p38MAPK signaling pathway on neuronal cells in rats with cerebral ischemia-reperfusion by changing the phosphorylation level of p38MAPK protein. Rats in CVP group had a significantly lower protein levels of Bax and higher protein levels of $\mathrm{Bcl}-2$ than those in CIRI and ANI group, indicating CVP alleviated the expressions of apoptosis proteins $(\mathrm{P}<0.05)$.

\section{Discussion}

CIRI can cause permanent damage to brain tissue during reperfusion of cerebral ischemia, often leading to necrotic nerve cells and apoptosis. It is therefore important to investigate possible methods of inhibiting nerve cell apoptosis and activating human endogenous repair for the recovery of nerve function in injured brain areas after cerebral ischemia-reperfusion. In this study, we found a protective effect of CVP similar to other herbal polysaccharides. We shed light on the treatment of CVP during IR injury. The results of this study demonstrated that CVP treatment: (I) decreased the expression of p38MAPK and Caspase- 3 by regulating the p38MAPK signaling pathway; (II) alleviated the CIRI-induced inflammatory response; (III) ultimately reduced the apoptosis of nerve cells, which had a therapeutic effect on CIRI rats. Numerous studies have found that p38MAPK is closely associated with apoptotic factors in ischemia-reperfusion injury tissue (11), which is also consistent with our findings.

CVP are a class of immunomodulators with a variety of biological functions. An analysis of the composition of CVP monosaccharide found that it is mainly composed of glucose, mannose, galactose, fucose, and xylose (12). CVP can regulate both the immune and redox processes (13). Considering that apoptosis is the final result of lipid peroxidation during cerebral ischemia and hypoxia, the effect of CVP on neuronal apoptosis during CIRI suggests that CVP may participate in cell death through a variety of apoptosis-related signals.

The MAPK family is a class of protein kinases that contain serine and threonine residues, and are widely distributed in the cytoplasm. They act by transmitting extracellular stimulatory signals to the nucleus, resulting in biological effects in the cells (14). The MAPK signaling pathway has several different subfamilies that can detect various stimuli outside the cell. Among them, p38MAPK is one of the signaling pathways closely related to apoptosis. In the nervous system, p38MAPK is mainly 
A

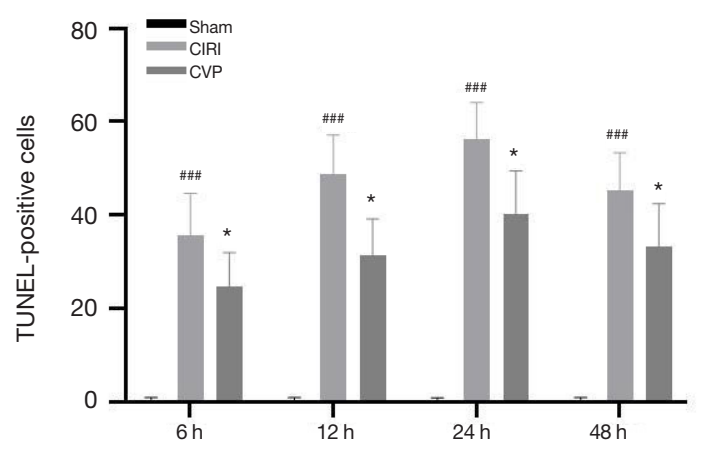

B
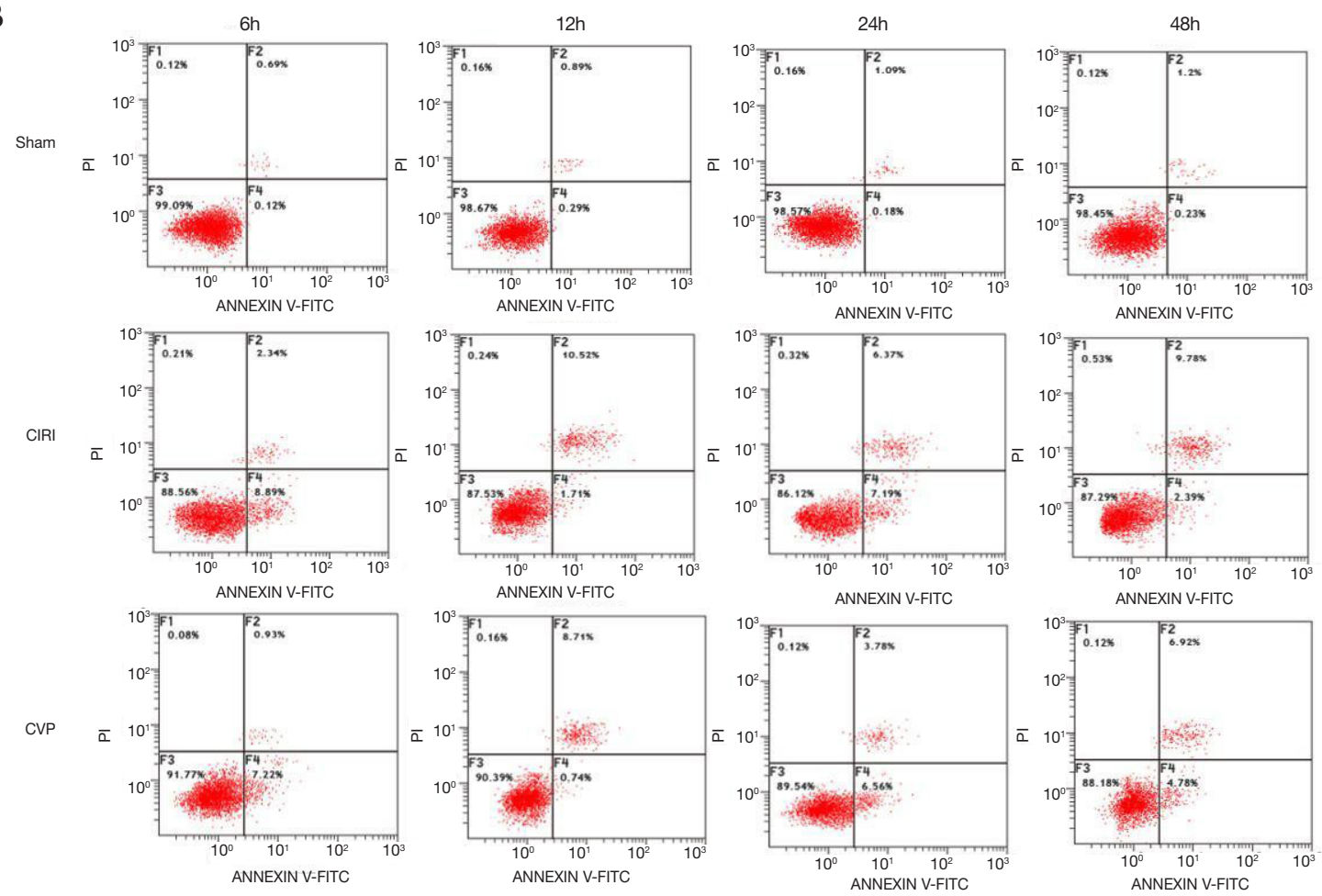

Figure 3 Effect of CVP on apoptotic cells in rats with cerebral ischemia-reperfusion at different time points. (A) Number of positive

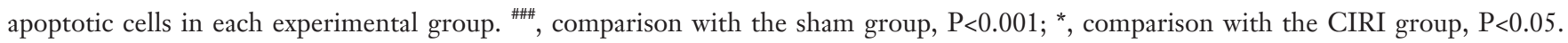
(B) Flow cytometry analysis of apoptotic cell rate in each experimental group. CIRI, cerebral ischemia-reperfusion injury; CVP, coriolus versicolor polysaccharides; ANI, anysomycin.

distributed in the nuclear region of neurons in the brain, regulating various physiological functions of the nervous system. In the presence of CIRI, the level of p38MAPK phosphorylation may stimulate Bax (a pro-apoptotic protein) to flow into the mitochondria, leading to a surge in neuronal apoptosis. This suggests that CIRI may be associated with the activation of the p38MAPK signaling pathway (15). Our results showed that the expression of p38MAPK and the apoptosis of nerve cells increased with time after CIRI, and so, p38MAPK and the number of apoptotic cells in CIRI rats were positively correlated. This observation confirmed that there is a close relationship 

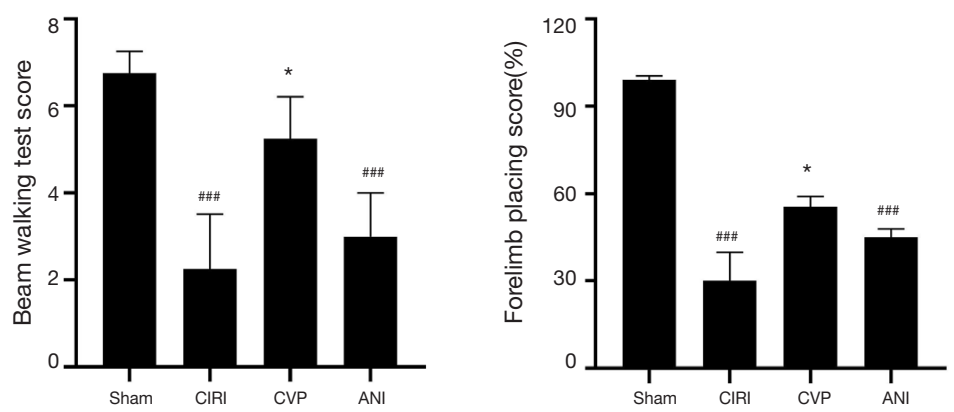

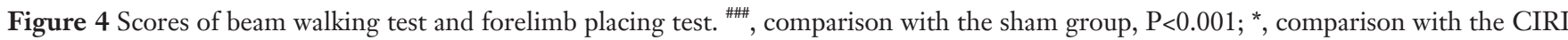
group, $\mathrm{P}<0.05$. CIRI, cerebral ischemia-reperfusion injury; CVP, Coriolus versicolor polysaccharides; ANI, anysomycin.

A

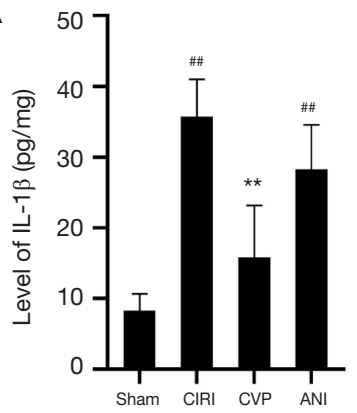

B

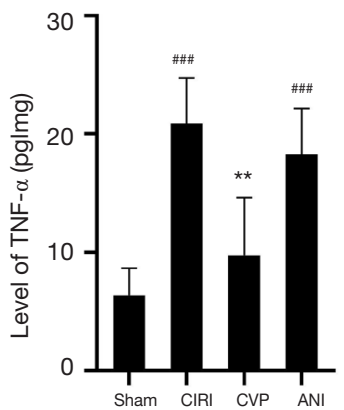

Figure 5 Expression of IL-1 $\beta$ and TNF- $\alpha$ in rats $24 \mathrm{~h}$ after cerebral ischemia-reperfusion. (A) Level of IL-10. \#\#, comparison with the

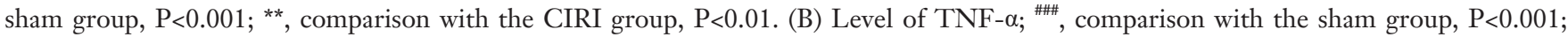
**, comparison with the CIRI group, $\mathrm{P}<0.01$. CIRI, cerebral ischemia-reperfusion injury; CVP, coriolus versicolor polysaccharides; IL-1 $\beta$, interleukin-1 $\beta$; TNF- $\alpha$, tumor necrosis factor- $\alpha$; ANI, anysomycin.

between p38MAPK and neuronal apoptosis (16). Recent studies have reported that in the early stage of cerebral ischemia-reperfusion, the activation of p38MAPK in ischemic brain tissue led to the expression of apoptotic genes and promoted further release of inflammatory factors such as TNF and interleukin (17). Moreover, inhibition of p38MAPK expression can suppress increases of these inflammatory factors. It is also well established that the inflammation caused by cerebral ischemia will increase the levels of IL- 1 and TNF- $\alpha$ in the ischemic area and stimulate the expression of $\mathrm{p} 38 \mathrm{MAPK}$. These observations indicate that p38MAPK plays an important role in CIRI.

In our study, treatment of CIRI with CVP decreased the levels of IL- $1 \beta$ and TNF- $\alpha$ in brain tissue, inhibited the expression of $\mathrm{p}-\mathrm{p} 38 \mathrm{MAPK}$, regulated the expression of $\mathrm{Bcl}-$ 2/Bax/Cleaved caspase- 3 apoptotic signaling pathways and significantly attenuated the apoptosis of nerve cells. Since cleaved-Caspase- 3 is a key protein in neuronal apoptosis during CIRI, inhibiting the expression of cleaved-Caspase-3 had a protective effect on nerve cells (18). Nevertheless, the p38 agonist anisomycin effectively reversed the protection role of CVP, suggesting CVP may offer neuroprotective effect through modulating p38MAPK signaling pathway.

In conclusion, our results suggest that CVP has a certain therapeutical effect on CIRI model rats, which establishes a theoretical foundation for further study. However, the specific mechanism through which CVP plays a role remains unclear. In future, we intend to further scrutinize the mechanisms of action of CVP macromolecular peptides in the intestinal tract and the brain, as well as the effective peptides that play a specific role in CVP. Further experimental analysis will provide a theoretical basis for the 
A

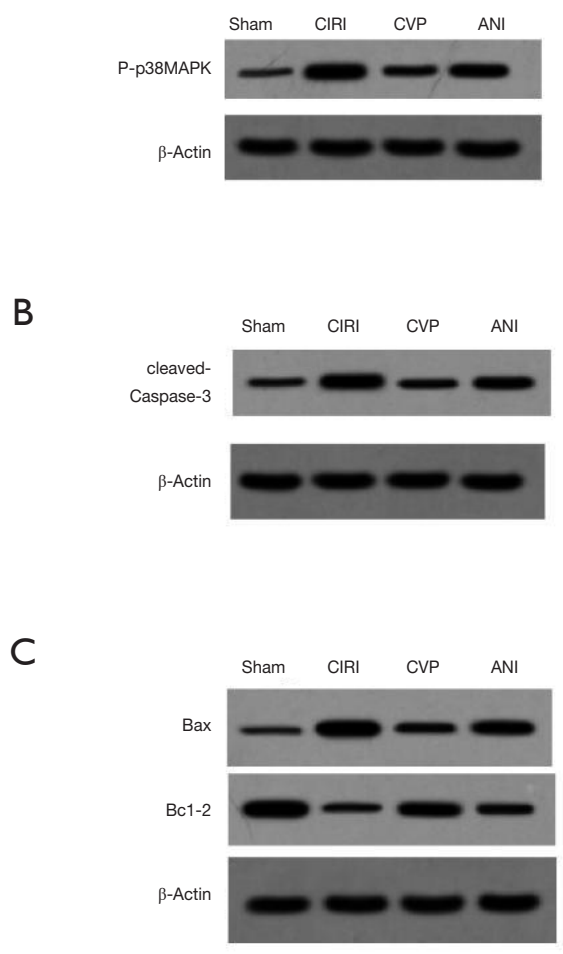

D
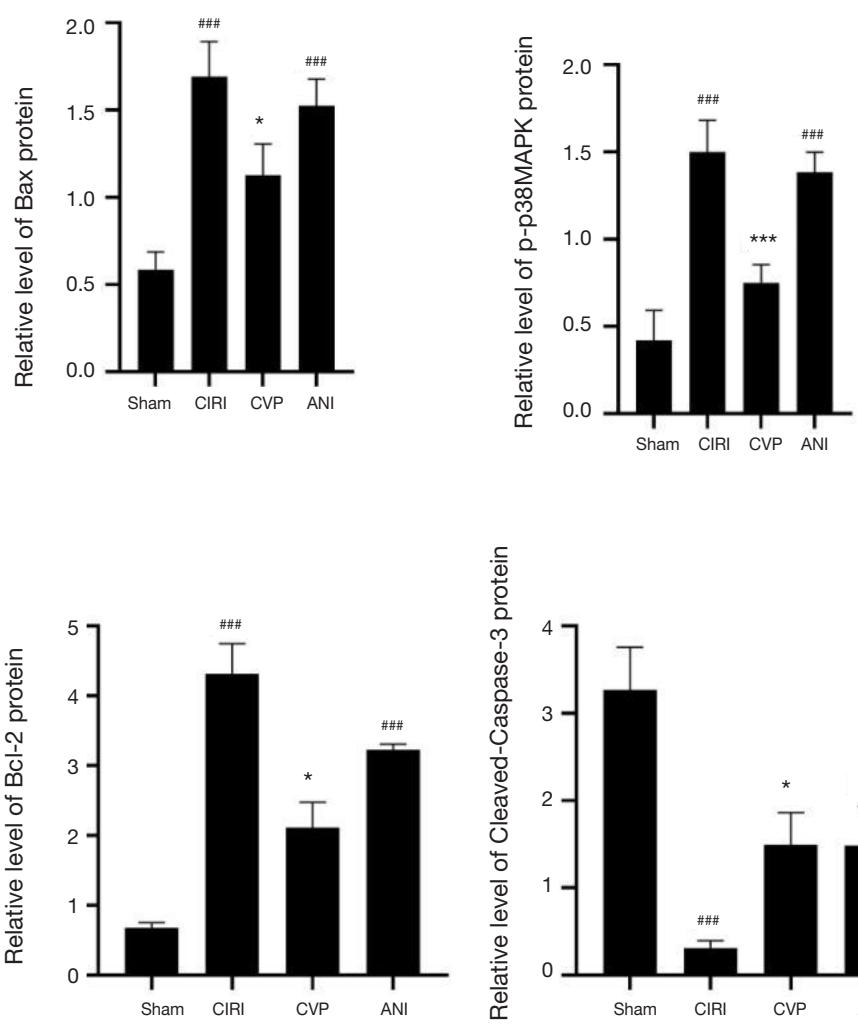

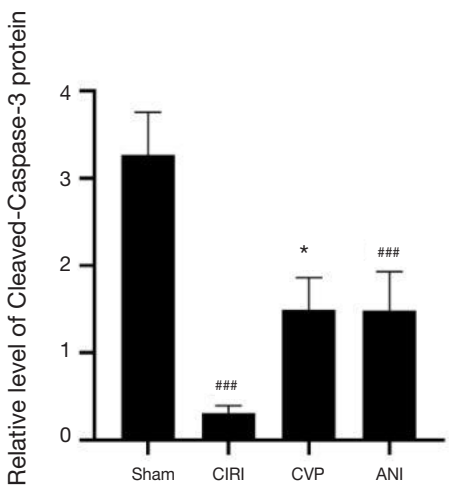

Figure 6 Expression of p38MAPK, p-p38MAPK, and cleaved-Caspase-3 protein in rats after $24 \mathrm{~h}$ of cerebral ischemia-reperfusion. (A,B,C) Western blot results of p-p38MAPK,cleaved-Caspase-3, Bax and Bcl-2. (D) Quantitative analysis of p-p38MAPK, cleaved-Caspase-3, Bax and $\mathrm{Bcl}-2$ protein levels. ${ }^{\# \#,}$, comparison with the sham group, $\mathrm{P}<0.001$; ${ }^{*}$, comparison with the CIRI group, $\mathrm{P}<0.05$; ${ }^{* * *}$, comparison with the CIRI group, $\mathrm{P}<0.001$; comparison with the CIRI group, $\mathrm{P}<0.05$. CIRI, cerebral ischemia-reperfusion injury; CVP, coriolus versicolor polysaccharides; p38MAPK, p38 mitogen-activated protein kinase; p-p38MAPK, phospho-p38MAPK; ANI, anysomycin.

study of CIRI treatment.

\section{Acknowledgments}

Funding: None.

\section{Footnote}

Reporting Checklist: The authors have completed the ARRIVE reporting checklist. Available at http://dx.doi. org/10.21037/atm-20-5759

Data Sharing Statement: Available at http://dx.doi. org/10.21037/atm-20-5759

Conflicts of Interest: All authors have completed the ICMJE uniform disclosure form (available at http://dx.doi. org/10.21037/atm-20-5759). The authors have no conflicts of interest to declare.

Ethical Statement: The authors are accountable for all aspects of the work in ensuring that questions related to the accuracy or integrity of any part of the work are appropriately investigated and resolved. This study was approved by Xinxiang Central Hospital (ZXYY-KY-0124). During our experiments, we strictly follow the Regulations on the Administration of Experimental Animals, The Guiding Opinions on treating Experimental Animals well and other relevant laws and regulations.

Open Access Statement: This is an Open Access article distributed in accordance with the Creative Commons Attribution-NonCommercial-NoDerivs 4.0 International License (CC BY-NC-ND 4.0), which permits the non- 
commercial replication and distribution of the article with the strict proviso that no changes or edits are made and the original work is properly cited (including links to both the formal publication through the relevant DOI and the license). See: https://creativecommons.org/licenses/by-nc-nd/4.0/.

\section{References}

1. Lakhan SE, Kirchgessner A, Hofer M. Inflammatory mechanisms in ischemic stroke: therapeutic approaches. J Transl Med 2009;7:97.

2. Li M, Qu YZ, Zhao ZW, et al. Astragaloside IV protects against focal cerebral ischemia/reperfusion injury correlating to suppression of neutrophils adhesion-related molecules. Neurochem Int 2012;60:458-65.

3. Shahreyar M, Bob-Manuel T, Khouzam RN, et al. Trends, predictors and outcomes of ischemic stroke and intracranial hemorrhage in patients with a left ventricular assist device. Ann Transl Med 2018;6:5.

4. Dong Q, Lin X, Shen L, et al. The protective effect of herbal polysaccharides on ischemia-reperfusion injury. Int J Biol Macromol. 2016;92:431-40.

5. Fisher M, Yang LX. Anticancer effects and mechanisms of polysaccharide-K (PSK): implications of cancer immunotherapy. Anticancer Res 2002;22:1737-54.

6. Kim BC, Kim YS, Lee JW, et al. Protective Effect of Coriolus versicolor Cultivated in Citrus Extract Against Nitric Oxide-Induced Apoptosis in Human Neuroblastoma SK-N-MC Cells. Exp Neurobiol 2011;20:100-9.

7. Chen J, Jin X, Zhang L, et al. A study on the antioxidant effect of Coriolus versicolor polysaccharide in rat brain tissues. Afr J Tradit Complement Altern Med 2013;10:481-4.

8. Ashraf MI, Ebner M, Wallner C, et al. A p38MAPK/ MK2 signaling pathway leading to redox stress, cell death and ischemia/reperfusion injury. Cell Commun Signal

Cite this article as: Li L, Li Y, Miao C, Liu Y, Liu R. Coriolus versicolor polysaccharides (CVP) regulates neuronal apoptosis in cerebral ischemia-reperfusion injury via the p38MAPK signaling pathway. Ann Transl Med 2020;8(18):1168. doi: 10.21037/atm-20-5759
2014;12:6.

9. Su D, Ma J, Zhang Z, et al. Protective Effects of UCF101 on Cerebral Ischemia-Reperfusion (CIR) is Depended on the MAPK/p38/ERK Signaling Pathway. Cell Mol Neurobiol 2016;36:907-14.

10. Longa EZ, Weinstein PR, Carlson S, et al. Reversible middle cerebral artery occlusion without craniectomy in rats. Stroke 1989;20:84-91.

11. Kobayashi M, Takeyoshi I, Yoshinari D, et al. P38 mitogen-activated protein kinase inhibition attenuates ischemia-reperfusion injury of the rat liver. Surgery 2002;131:344-9.

12. Qin XD, Liu JK. Chemical constituents of Coriolus versicolor. Journal of Yunnan Agricultural University 2012;27:774-6.

13. Kim HM, Han SB, Oh GT, et al. Stimulation of humoral and cell mediated immunity by polysaccharide from mushroom Phellinus linteus. Int J Immunopharmacol 1996;18:295-303.

14. Lee JC, Laydon JT, McDonnell PC, et al. A protein kinase involved in the regulation of inflammatory cytokine biosynthesis. Nature 1994;372:739-46.

15. Thornton TM, Pedraza-Alva G, Deng B, et al. Phosphorylation by 38 MAPK as an alternative pathway for GSK3 beta inactivation. Science 2008;320:667-70.

16. Yun H, Kim HS, Lee S, et al. AMP kinase signaling determines whether c-Jun $\mathrm{N}$-terminal kinase promotes survival or apoptosis during glucose deprivation. Carcinogenesis 2009;30:529-37.

17. Rossi DJ, Brady JD, Mohr C. Astrocyte metabolism and signaling during brain ischemia. Nat Neurosci 2007;10:1377-86.

18. Robinson MJ, Cobb MH. Mitogen-activated protein kinase pathways. Curr Opin Cell Biol 1997;9:180-6.

(English Language Editor: A. Kassem) 